\title{
AN EXTENSION OF THE HARDY-LITTLEWOOD INEQUALITY
}

\author{
M. K. KWONG AND A. ZETTL ${ }^{1}$
}

ABstract. The Hardy-Littlewood inequality is extended from $L^{2}$ to $L_{w}^{2}$ where $w$ is any positive nondecreasing function.

In this note we establish the inequality

$$
\left(\int_{J}\left|y^{\prime}\right|^{2} w\right)^{2} \leqslant 4 \int_{J}|y|^{2} w \int_{J}\left|y^{\prime \prime}\right|^{2} w
$$

for any complex valued function $y$ satisfying

$$
y \in L_{w}^{2}(J), \quad y^{\prime} \text { locally absolutely continuous, } \quad y^{\prime \prime} \in L_{w}^{2}(J) .
$$

Here $J=(0, \infty)$ or $J=(-\infty, \infty)$ and $w$ is any positive nondecreasing function on $J$. The case $w(t) \equiv 1$ is the inequality of the title; in this case the constant 4 is best possible when $J=(0, \infty)$ but can be replaced by 1 when $J=(-\infty, \infty)[3]$.

Proof. Suppose $y$ satisfies (2). First we show that

$$
y^{2}(t) w(t) \rightarrow 0 \text { as } t \rightarrow \infty .
$$

Since $w(t)$ is nondecreasing we have

$$
w(t) \int_{t}^{\infty}\left|y^{(i)}\right|^{2} \leqslant \int_{t}^{\infty}\left|y^{(i)}\right|^{2} w, \quad i=0,2 .
$$

Hence $y$ and $y^{\prime \prime}$ are in $L^{2}(t, \infty)$ and by a well-known result [2] relating the supremum norm of $y^{\prime}$ to the $L^{2}$ norms of $y$ and $y^{\prime \prime}$ we have

$$
\begin{aligned}
|y(t)|^{2} & \leqslant\|y\|_{\infty,(t, \infty)}^{2} \leqslant K\left(\int_{t}^{\infty}|y|^{2}\right)^{1 / 4}\left(\int_{t}^{\infty}\left|y^{\prime \prime}\right|^{2}\right)^{3 / 4} \\
& \leqslant K w^{-1}(t)\left(\int_{t}^{\infty}|y|^{2} w\right)^{1 / 4}\left(\int_{t}^{\infty}\left|y^{\prime \prime}\right|^{2} w\right)^{3 / 4} .
\end{aligned}
$$

Therefore $w(t) y^{2}(t) \rightarrow 0$ as $t \rightarrow \infty$.

Now we show that the differentiation operator $A$ defined by $A y=y^{\prime}$ is dissipative on $L_{w}(0, \infty)$. We have, for $y=u+i v$,

$$
\operatorname{Re}(A y, y)=\int_{0}^{\infty}\left(u^{\prime} u+v^{\prime} v\right) w
$$

Received by the editors October 23, 1978.

AMS (MOS) subject classifications (1970). Primary 47A30; Secondary 47B25.

Key words and phrases. Landau, Hardy-Littlewood inequality, norm inequality for derivatives.

${ }^{1}$ The research of this author was partially supported by NSF grant MCS76-06623 A01. 
and

$$
2 \int_{0}^{t} u^{\prime} u w=u^{2}(t) w(t)-u^{2}(0) w(0)-\int_{0}^{t} u^{2} d w .
$$

From (3) and (5) we conclude that $\operatorname{Re}(A y, y) \leqslant 0$ for all $y$ satisfying (2), i.e., $y$ in the domain of $A^{2}$ on $L_{w}^{2}(0, \infty)$ and hence $A$ is dissipative. Since every dissipative operator on Hilbert space has an $m$-dissipative extension [1] inequality (1) follows from Kato's inequality for $m$-dissipative operators [4]. The proof for $J=(-\infty, \infty)$ is entirely similar.

Inequality (1) does not hold for arbitrary weight functions $w$ as can be seen from the simple example: $y(t)=t, w(t)=e^{-t}, 0 \leqslant t<\infty$.

Kato in [4] also characterizes the cases of equality. From this characterization follows that there is equality in (1) if and only if

$$
\int_{J} f_{b, c} f_{b, c}^{\prime \prime} w=0
$$

for some constants $b, c$, where

$$
f_{b, c}(t)=\exp (-b t / 2) \sin (\sqrt{3} b t / 2-c) \text {. }
$$

When $w=1$ and $J=(0, \infty)$, all extremals of (1) are given by $a f_{b, c}$ with $c=\pi / 3$ and $a, b$ constants with $b>0$. It can be seen from (6) that there are nonconstant weight functions $w$ for which (1) has extremals yielding the best constant 4 in both cases $J=(0, \infty)$ and $J=(-\infty, \infty)$. For instance $w(t)=1$ in $[0,4 \pi / \sqrt{3}]$ and $w(t)=2$ for $t>4 \pi / \sqrt{3}$ has extremal

$$
f(t)=\exp (-t / 2) \sin (\sqrt{3} t / 2-\pi / 3)
$$

giving the constant 4 in (1). Of course when (6) does not hold the best constant in (1) may be less than 4 .

\section{REFERENCES}

1. R. Beals, Topics in operator theory, Univ. of Chicago Press, Chicago, Ill., 1971.

2. V. N. Gabusin, Inequalities for norms of a function and its derivatives in $L^{p}$ metrics, Mat. Zametki 1 (1967), 291-298. (Russian)

3. G. H. Hardy, J. E. Littlewood and G. Polyá, Inequalities, Cambridge Univ. Press, Cambridge, 1952.

4. T. Kato, On an inequality of Hardy, Littlewood and Polyá, Advances in Math. 7 (1971), 217-218.

Department of Mathematical Sciences, Northern Illinois University, DeKalb, Illinois 60115 